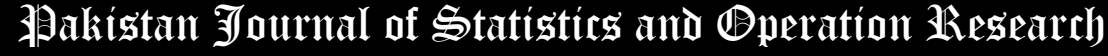

\section{Extended Poisson Lomax Distribution}

\author{
M. S. Hamed ${ }^{1,2^{*}}$ \\ * Corresponding Author
}

1. Management Information System Department, Taibah University, Saudi Arabia, moswilem@gmail.com

2. Department of Statistics, Mathematics and Insurance, Benha University, Egypt

\begin{abstract}
The main goal of this article is to introduce a new extension of the continuous Lomax distribution with a strong physical motivation. Some of its statistical properties such as moments, incomplete moments, moment generating function, quantile function, random number generation, quantile spread ordering and moment of the reversed residual life are derived. Two applications are provided to illustrate the importance and flexibility of the new model.
\end{abstract}

Key Words: Maximum Likelihood Estimation; Quantile Function; Generating Function; Moments; Zero Truncated Poisson.

Mathematical Subject Classification: 62N01; 62N02; 62E10.

\section{Introduction and physical motivation}

In this work we introduce a new extension of the Lomax (Lx) distribution by compounding the Burr X Lomax (BrXLx) model with the zero truncated Poisson (ZTP) distribution. A random variable (r.v.) $T$ is said to have the BrXLx distribution (see Yousof et al. (2017) and Abouelmagd (2018)) if its cumulative distribution function (CDF) is given

$$
H_{B r X L x}^{(\theta, \alpha) \beta}(t)=\left[1-\exp \left(-\left\{\left[1+\left(t \beta^{-1}\right)\right]^{\alpha}-1\right\}^{2}\right)\right]^{\theta},
$$

where the CDF and probability density function (PDF) (for $x>0$ ) of the Lx distribution (see Lomax (1954)) with positive parameters $\alpha$ and $\beta$ are $G_{L x}^{(\alpha, \beta)}(x)=\left\{1-\left[1+\left(x \beta^{-1}\right)\right]^{-\alpha}\right\}$ and $g_{L x}^{(\alpha, \beta)}(x)=\alpha \beta^{-1}\left[1+\left(x \beta^{-1}\right)\right]^{-\alpha-1}$, respectively. Suppose that a system has $N$ subsystems functioning independently at a given time where $N$ has ZTP distribution with parameter $\lambda$, then the conditional probability distribution of a Poisson-distributed random variable (r.v.), given that the value of the r.v. is not zero. The probability mass function (PMF) of $N$ is given by

$$
P M F_{Z T P}^{(\lambda)}(N \mid N=n)=\left.\frac{\exp (-\lambda) \lambda^{n}}{n ! a_{\lambda}}\right|_{(n=1,2, \ldots)},
$$

where $a_{\lambda}=[-\exp (-\lambda)+1]$. Note that for ZTP r.v., the expected value $E(N \mid \lambda)$ and variance $\operatorname{Var}(N \mid \lambda)$ are respectively given by $E(N \mid \lambda)=\lambda / a_{\lambda}$ and $\operatorname{Var}(N \mid \lambda)=\frac{\lambda}{a_{\lambda}}\left\{-\frac{\lambda}{a_{\lambda}}+(1+\lambda)\right\}$. Suppose that the failure time of each subsystem has the BrXLx. Let $Y_{i}$ denote the failure time of the $i^{\text {th }}$ subsystem and let $X=\min \left\{Y_{1}, Y_{2}, \cdots, Y_{N}\right\}$, then the conditional CDF of $X$ given $N$ via (1) is

$$
F(x \mid N)=1-\operatorname{Pr}(X>x \mid N)=-\left[1-H_{B r X L x}^{(\theta, \alpha, \beta)}(x)\right]^{N}+1 .
$$

Following Ramos et al. (2015), Aryal and Yousof (2017), Sen et al. (2018), Korkmaz et al. (2018) and Yousof et al. (2020), the unconditional CDF of the ZTPBrLx density function can be written as

$$
F(x)=\frac{1}{a_{\lambda}}\left(1-\exp \left\{-\lambda\left[1-\exp \left(-\left\{\left[1+\left(t \beta^{-1}\right)\right]^{\alpha}-1\right\}^{2}\right)\right]^{\theta}\right\}\right),
$$

with corresponding PDF as

$$
f(x)=\frac{2 \theta \lambda \alpha\left\{1-\left[1+\left(x \beta^{-1}\right)\right]^{-\alpha}\right\} \exp \left[-\left\{\left[1+\left(t \beta^{-1}\right)\right]^{\alpha}-1\right\}^{2}\right]\left\{1-\exp \left[-\left\{\left[1+\left(t \beta^{-1}\right)\right]^{\alpha}-1\right\}^{2}\right]\right\}^{\theta-1}}{\beta a_{\lambda}\left[1+\left(x \beta^{-1}\right)\right]^{1-2 \alpha} \exp \left(\lambda\left\{1-\exp \left[-\left\{\left[1+\left(t \beta^{-1}\right)\right]^{\alpha}-1\right\}^{2}\right]\right\}^{\theta}\right)}
$$


The plots of the ZTPBrXLx PDF and HRF are displayed in Figures 1 and 2 for selected parameter values. From Figures 1 and 2 we conclude that the ZTPBrXLx distribution will be suitable in modeling right skewed, symmetric and unimodal data sets. The HRF of the ZTPBrXLx may have the increasing, decreasing, $\mathbf{J}$ shapes, unimodal, batube and constant shapes.
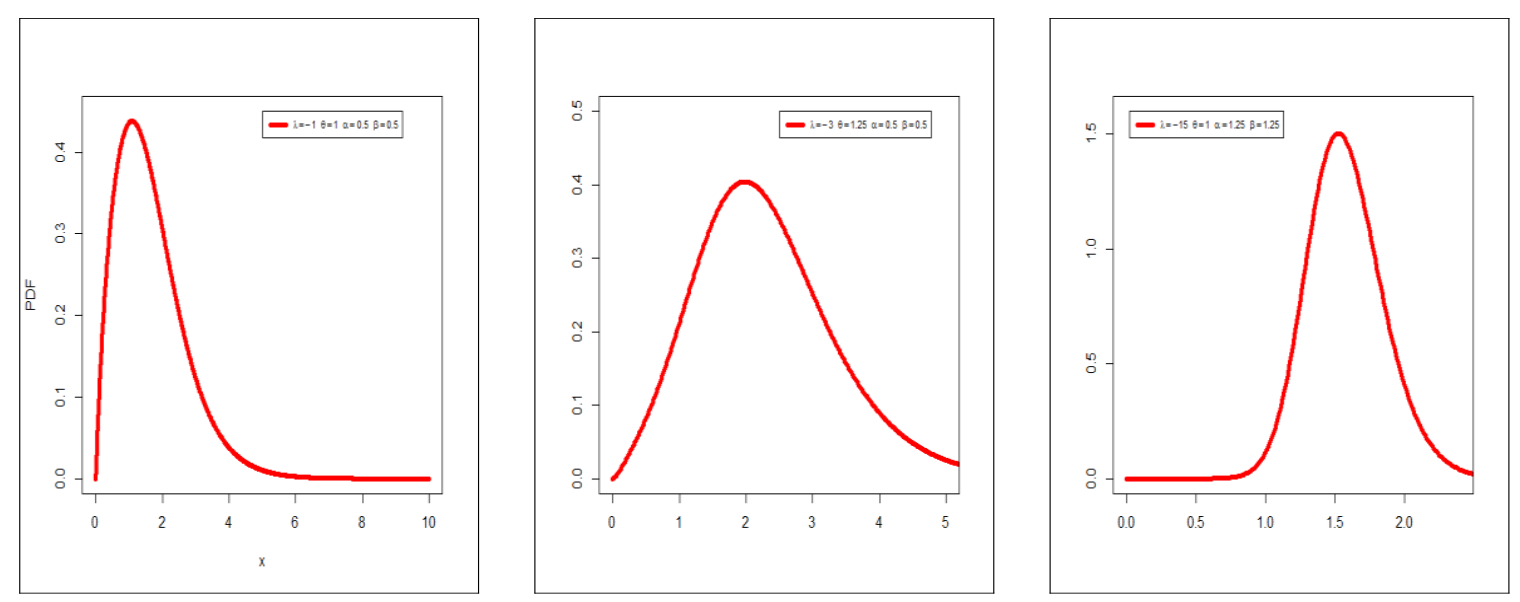

Figure 1: some PDF plots for the ZTPBrXLx model.
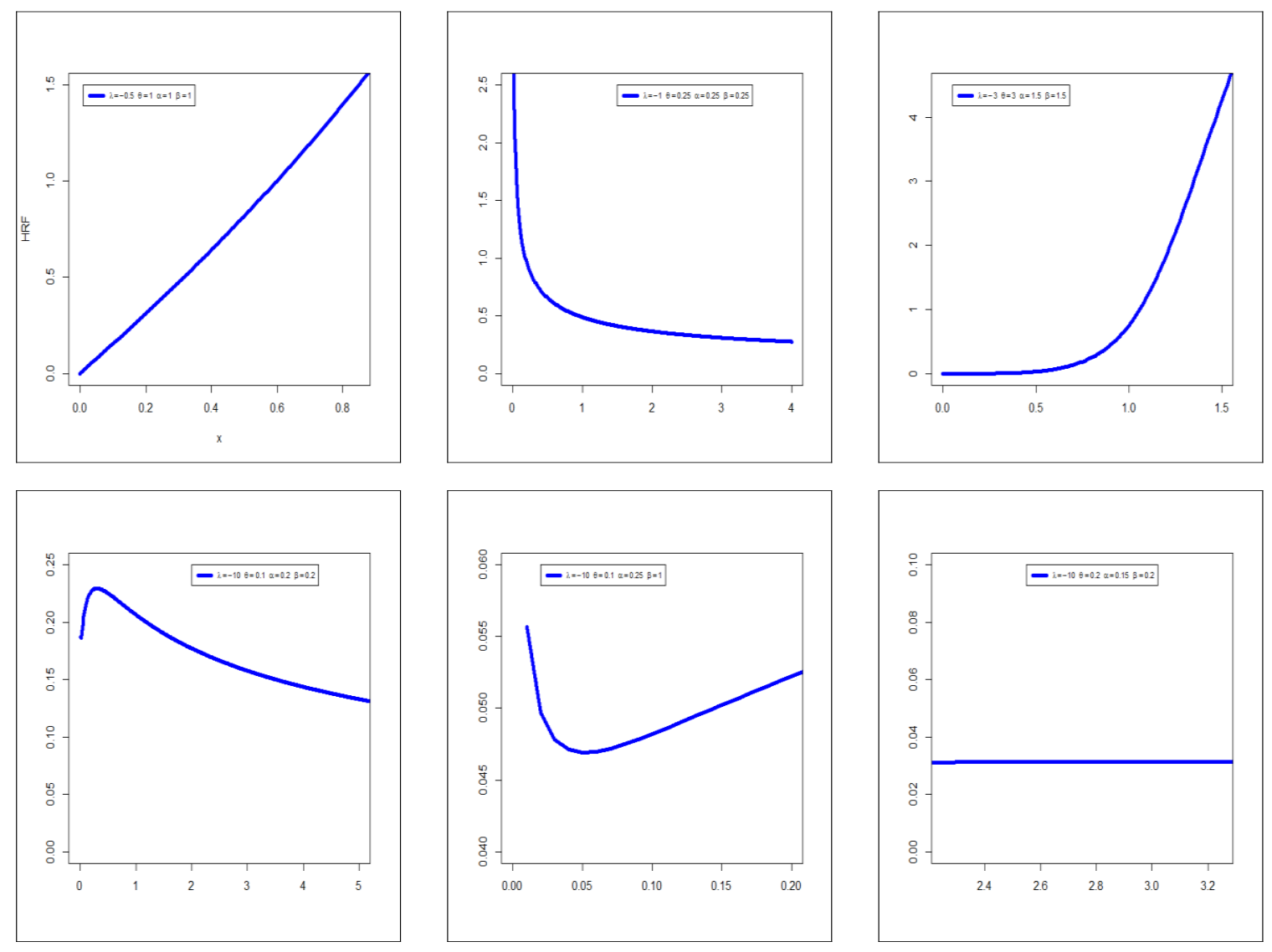

Figure 2: some HRF plots for the ZTPBrXLx model. 
This rest of this work is organized as follows: In Section 2, we derive some properties of the new model. Maximum likelihood estimation for the unknown model parameters under uncensored case is addressed in Section 3. In Sections 4 , the potentiality of the proposed model is illustrated by means of two real data sets. Finally, Section 5 provides some concluding remarks.

\section{Mathematical properties \\ 2.1 Useful expansions \\ Consider the power series}

$$
\exp (k)=\sum_{m=0}^{\infty}\left(k^{m} / m !\right)
$$

using (6), the PDF in (5) can be expanded as

$$
\begin{aligned}
f(x)=\frac{2 \theta \alpha \beta^{-1}\left\{1-\left[1+\left(x \beta^{-1}\right)\right]^{-\alpha}\right\}}{\left[1+\left(x \beta^{-1}\right)\right]^{1+\alpha}\left\{\left[1+\left(x \beta^{-1}\right)\right]^{-\alpha}\right\}^{3}} \exp \left[-\left\{\left[1+\left(t \beta^{-1}\right)\right]^{\alpha}-1\right\}^{2}\right] \\
\quad \times \sum_{h=0}^{\infty} \frac{\lambda^{1+h}(-1)^{h}}{h ! a_{\lambda}} \underbrace{\left\{1-\exp \left[-\left\{\left[1+\left(t \beta^{-1}\right)\right]^{\alpha}-1\right\}^{2}\right]\right\}^{\theta(1+h)-1}}_{A} .
\end{aligned}
$$

Consider the following power series

$$
(1-q)^{p-1}=\left.\sum_{h=0}^{\infty}\left\{\left[(-1)^{h} \Gamma(p)\right] /[h ! \Gamma(p-h)]\right\} q^{h}\right|_{(|q|<1 \text { and } p>0 \text { is a realnon-integer })} .
$$

Applying (8) to the quantity $A$ in Equation (7) we have

$$
\begin{aligned}
f(x) & =\frac{2 \theta \alpha \beta^{-1}\left[1+\left(x \beta^{-1}\right)\right]^{-\alpha-1}\left\{1-\left[1+\left(x \beta^{-1}\right)\right]^{-\alpha}\right\}}{a_{\lambda}\left\{\left[1+\left(x \beta^{-1}\right)\right]^{-\alpha}\right\}^{3}} \sum_{h, i=0}^{\infty} \frac{\lambda^{1+h}(-1)^{h+i} \Gamma(\theta(1+h))}{i ! \Gamma(\theta(1+h)-i)} \\
& \times \underbrace{\exp \left[-(1+i)\left\{\left[1+\left(t \beta^{-1}\right)\right]^{\alpha}-1\right\}^{2}\right]}_{B} .
\end{aligned}
$$

Applying the power series to the quantity $B$, Equation (9) becomes

$f(x)=\sum_{h, i, j=0}^{\infty} \frac{2 \theta \lambda^{1+h} \alpha \beta^{-1}(-1)^{h+i+j}(1+i)^{j} \Gamma(\theta(1+h))}{i ! j !\left(1-e^{-\lambda}\right) \Gamma(\theta(1+h)-i)} \frac{\left[1+\left(x \beta^{-1}\right)\right]^{-\alpha-1}\left\{1-\left[1+\left(x \beta^{-1}\right)\right]^{-\alpha}\right\}^{2 j+1}}{\underbrace{\left\{\left[1+\left(x \beta^{-1}\right)\right]^{-\alpha}\right\}^{3+2 j}}_{c}}$.

Consider the series expansion

$$
(1-q)^{-p}=\left.\sum_{t=0}^{\infty}\left\{\left[\Gamma(p+t) q^{t}\right] /[t ! \Gamma(p)]\right\}\right|_{(|q|<1, p>0)} .
$$

Applying (11) to (10) for the quantity $C$, Equation (10) becomes

where

$$
f(x)=\sum_{j, k=0}^{\infty} \zeta_{j, k} h_{[2(1+j)+k]}(x ; \alpha, \beta)
$$

and

$$
\zeta_{j, k}=\sum_{h, i=0}^{\infty} \frac{2 \theta \lambda^{1+h}(-1)^{j+h+i}(1+i)^{j} \Gamma(\theta(1+h)) \Gamma(3+2 j+k)}{j ! k ! i ! a_{\lambda} \Gamma(\theta(1+h)-i) \Gamma(3+2 j)[2(1+j)+k]},
$$

$$
h_{[2(1+j)+k]}(x ; \alpha, \beta)=[2(1+j)+k] \alpha \beta^{-1}\left[1+\left(x \beta^{-1}\right)\right]^{-\alpha-1}\left\{1-\left[1+\left(x \beta^{-1}\right)\right]^{-\alpha}\right\}^{2 j+k+1},
$$

is the PDF of the exp-Lx model with power parameter $[2(1+j)+k]$. Equation (12) reveals that the density of $X$ can be expressed as a linear mixture of exp-Lx densities. So, several mathematical properties of the new model can be obtained from those of the exp-Lx distribution. Similarly, the CDF of the ZTPBrXLx model can also be expressed as a mixture of exp-Lx CDFs given by

$$
F(x)=\sum_{j, k=0}^{\infty} \zeta_{j, k} H_{[2(1+j)+k]}(x ; \alpha, \beta),
$$

where

$$
H_{2(1+j)+k}(x ; \alpha, \beta)=\left\{1-\left[1+\left(t \beta^{-1}\right)\right]^{-\alpha}\right\}^{2(1+j)+k},
$$

is the $\mathrm{CDF}$ of the exp-Lx model with power parameter $[2(1+j)+k]$. 
Theorem Let $Y$ be a continuous r.v. having the exp-Lx model with power parameter $\delta$, then the $q$ th ordinary and incomplete moments $\left(\mu_{q}^{\prime}(y)\right.$ and $\left.m_{q}(y)\right)$ of the exp-Lx r.v. (defined in this subsection) given by

and

$$
\mu_{q}^{\prime}(y)=\left.\sum_{w=0}^{q} \delta \beta^{q}(-1)^{w}\left(\begin{array}{c}
q \\
w
\end{array}\right) B\left(\delta, \frac{w-q}{\alpha}+1\right)\right|_{(\alpha>q)},
$$

where

$$
m_{q}(y)=\left.\sum_{w=0}^{q} \delta \beta^{q}(-1)^{w}\left(\begin{array}{c}
q \\
w
\end{array}\right) B_{t}\left(\delta, \frac{w-q}{\alpha}+1\right)\right|_{(\alpha>q)},
$$

is the complete beta function and

$$
\left.B\left(c_{1}, c_{2}\right)\right|_{\left[c_{1}, c_{2} \notin(0,-1,-2, \ldots)\right]}=\int_{0}^{1} w^{c_{1}-1}(1-w)^{c_{2}-1} d w=\Gamma\left(c_{1}\right) \Gamma\left(c_{2}\right) / \Gamma\left(c_{1}+c_{2}\right),
$$

$$
\left.B_{z}\left(c_{1}, c_{2}\right)\right|_{\left[c_{1}, c_{2} \notin(0,-1,-2, \ldots)\right]}=\int_{0}^{z} w^{c_{1}-1}(1-w)^{c_{2}-1} d w=\sum_{m=0}^{\infty} \frac{\left(1-c_{2}\right)_{j}}{m !\left(c_{1}+m\right)} z^{c_{1}+m},
$$

is the incomplete beta function.

\subsection{Moments and incomplete moments}

The $r^{\text {th }}$ ordinary moment of $X$, say $\mu_{r}^{\prime}$, follows from (12) as

where

$$
\mu_{r}^{\prime}=E\left(X^{r}\right)=\left.\sum_{j, k=0}^{\infty} \sum_{w=0}^{r} \zeta_{j, k, w}^{\{[2(1+j)+k], r\}} B\left([2(1+j)+k], 1+\frac{w-r}{\alpha}\right)\right|_{(\alpha>r)},
$$

The $r^{\text {th }}$ incomplete moment of $X$ is defined by

$$
\zeta_{j, k, w}^{\{[2(1+j)+k], r\}}=\zeta_{j, k}[2(1+j)+k] \beta^{r}(-1)^{w}\left(\begin{array}{l}
r \\
w
\end{array}\right) .
$$

$$
m_{r}(y)=\int_{-\infty}^{y} x^{r} f(x) d x .
$$

We can write from (12)

$$
m_{r}(x)=\left.\sum_{j, k=0}^{\infty} \sum_{w=0}^{r} \zeta_{j, k, w}^{\{[2(1+j)+k], r\}} B_{t}\left([2(1+j)+k], 1+\frac{w-r}{\alpha}\right)\right|_{(\alpha>r)}
$$

Setting $r=1$ in (14) gives the mean of $X$. Two important applications of the first incomplete moment $\left.m_{r}(y)\right|_{(r=1)}$ are related to the mean deviations about the mean and median and to the Bonferroni and Lorenz curves.

\subsection{Generating function}

The moment generating function (mgf) of $X$, say $M(t)=E(\exp (t X))$, is obtained from (12) as

$$
M(t)=\left.\sum_{j, k, r=0}^{\infty} \sum_{w=0}^{r} \frac{t^{r}}{r !} \zeta_{j, k, w}^{\{[2(1+j)+k], r\}} B\left([2(1+j)+k], 1+\frac{w-r}{\alpha}\right)\right|_{(\alpha>r)} .
$$

\subsection{Quantile function and random number generation}

The quantile function (qf) of $X$, where $X \sim \operatorname{ZTPBrXLx}(\lambda, \theta, \alpha, \beta)$, is obtained by inverting (4) as

$$
Q(u)=\beta\left(-\left\{-\ln \left[1-\left(\frac{-\ln \left\{1-u a_{\lambda}\right\}}{\lambda}\right)^{\frac{1}{\theta}}\right]\right\}^{\frac{1}{2}}\right)^{-\frac{1}{\alpha}}
$$

so, simulating the ZTPBrXLx r.v. is straightforward. If $U$ is a uniform variate on the unit interval, $(0,1)$, then the random variable $X=Q(u)$ follows (5).

\subsection{Quantile spread ordering}

The quantile spread (QS) of the r.v. $Z \sim \operatorname{ZTPBrXLx}(\lambda, \theta, \alpha, \beta)$ having $\operatorname{CDF}(5)$ is given by 


$$
[Q S]_{X}(\pi)=\left.\left\{\left[F^{-1}(\pi)\right]-\left[F^{-1}(1-\pi)\right]\right\}\right|_{(\pi \in(0.5,1))}
$$

which also implies that $[Q S]_{X}(\pi)=\left[S^{-1}(1-\pi)\right]-\left[S^{-1}(\pi)\right]$ where $F^{-1}(\pi)=S^{-1}(1-\pi)$ and $S(z)=1-F(z)$, is the survival function (SF). The QS of a distribution describes how the probability mass is placed symmetrically about its median and hence can be used to formalize concepts such as "peakedness" and "tail weight traditionally associated with kurtosis". So, it allows us to separate concepts of "kurtosis" and "peakedness" for asymmetric models. Let $Z_{1}$ and $Z_{2}$ be two r.v.s following the $\operatorname{ZTPBrXLx}(\lambda, \theta, \alpha, \beta)$ with quantile spreads $[Q S]_{Z_{1}}$ and $[Q S]_{Z_{2}}$, respectively. Then $Z_{1}$ is called smaller than $Z_{2}$ in quantile spread order, denoted by $Z_{1} \leq_{[Q S]} Z_{2}$, if $[Q S]_{Z_{1}}(\pi) \leq$ $\left.[Q S]_{Z_{2}}(\pi)\right|_{(\pi \in(0.5,1))}$.

The following properties of the QS order can be obtained:

$1-$ if $Z_{2}$ and $Z_{1}$ have the same distribution then $[Q S]_{Z_{2}}=[Q S]_{Z_{1}}$.

2 - The order $\leq_{[Q S]}$ is location-free if $\left.\left[\left(Z_{1}+c\right) \leq_{[Q S]} Z_{2}\right]\right|_{\left(c \in R^{+}\right)}$then $Z_{1} \leq_{[Q S]} Z_{2}$.

3 - The order $\leq_{[Q S]}$ is dilative $Z_{1} \leq_{[Q S]} c Z_{1}$ whenever $c \geq 1$ and $Z_{2} \leq\left._{[Q S]} c Z_{2}\right|_{(c \geq 1)}$.

4 - Let $F_{Z_{1}}$ and $F_{Z_{2}}$ be symmetric, then $Z_{1} \leq_{[Q S]} Z_{2}$ iff $F_{Z_{1}}^{-1}(\pi) \leq\left. F_{Z_{2}}^{-1}(\pi)\right|_{(\pi \in(0.5,1))}$.

5 - The order $\leq_{[Q S]}$ implies ordering of the mean absolute deviation around the median, say MAD

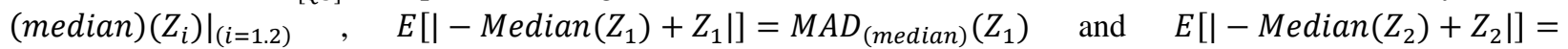
$M A D_{\text {(median) }}\left(Z_{2}\right)$, where $Z_{1} \leq_{[Q S]} Z_{2} \Rightarrow M A D_{(\text {median })}\left(Z_{1}\right) \leq_{[Q S]} M A D_{(\text {median })}\left(Z_{2}\right)$. Finally $Z_{1} \leq_{[Q S]} Z_{2}$ iff $Z_{1} \leq_{[Q S]}-Z_{2}$.

\subsection{Moment of the reversed residual life}

The $n^{t h}$ moment of the reversed residual life, say $\Upsilon_{n}(t)=\left.E\left[(t-X)^{n}\right]\right|_{(X \leq t, t>0)} ^{(n=1,2, \ldots)}$, uniquely determines the CDF, the we have $\Upsilon_{n}(t)=F^{-1}(t) \int_{0}^{t}(t-x)^{n} d F(x)$. The $n^{t h}$ moment of the reversed residual life of $X$ becomes

where

$$
\Upsilon_{n}(t)=\left.F^{-1}(t) \sum_{j, k=0}^{\infty} \sum_{w=0}^{n} \sum_{r=0}^{n} \xi_{j, k, w, r}^{\{[2(1+j)+k], n\}} B_{t}\left([2(1+j)+k], \frac{w-n}{\alpha}+1\right)\right|_{(\alpha>n)},
$$

$$
\xi_{j, k, w, r}^{\{[2(1+j)+k], n\}}=\zeta_{j, k}(-1)^{r} t^{n-r}[2(1+j)+k] \beta^{n}(-1)^{w}\left(\begin{array}{c}
n \\
w
\end{array}\right)\left(\begin{array}{l}
n \\
r
\end{array}\right)
$$

\section{Estimation}

We will consider the method of the maximum likelihood to estimation the unknown parameters $(\lambda, \theta, \alpha, \beta)$ of the new model from the complete samples. Let $x_{1}, \cdots, x_{n}$ be a random sample (r.s.) from the ZTPBrXLx models with parameter vector $\Phi=(\lambda, \theta, \alpha, \beta)^{T}$, the log-likelihood function for $\Phi$ is given by

$$
\begin{gathered}
\ell_{n}(\Phi)=n \log 2+n \log \theta+n \log \lambda+n \log \alpha-n \log \beta-n \log a_{\lambda} \\
+(\theta-1) \sum_{i=1}^{n} \log \left\{1-\exp \left[-\left(\left[1+\left(x_{i} \beta^{-1}\right)\right]^{\alpha}-1\right)^{2}\right]\right\} \\
-(\alpha+1) \sum_{i=1}^{n} \log \left[1+\left(x_{i} \beta^{-1}\right)\right]+\sum_{i=1}^{n} \log \left\{1-\left[1+\left(x_{i} \beta^{-1}\right)\right]^{-\alpha}\right\}-3 \sum_{i=1}^{n} \log \left\{\left[1+\left(x_{i} \beta^{-1}\right)\right]^{-\alpha}\right\} \\
-\sum_{i=1}^{n}\left(\left[1+\left(x_{i} \beta^{-1}\right)\right]^{\alpha}-1\right)^{2}-\lambda \sum_{i=1}^{n}\left\{1-\exp \left[-\left(\left[1+\left(x_{i} \beta^{-1}\right)\right]^{\alpha}-1\right)^{2}\right]\right\}^{\theta} .
\end{gathered}
$$

The above log-likelihood function can be maximized numerically by via R (optim) or SAS (PROC NLMIXED) or Ox program (sub-routine MaxBFGS), among others. For interval estimation of the parameters, the elements of the $4 \times 4$ observed information matrix $J(\Phi)$ can be derived and evaluated numerically (see section 4).

\section{Modeling data}

In this section, we provide two applications to show empirically the potentiality of the ZTPBrXLx model. In order to compare the fits of the ZTPBrXLx distribution with other competing distributions, we consider the Cramér-von Mises $\left(W^{*}\right)$ and the Anderson-Darling $\left(A^{*}\right)$ statistics. These two statistics are widely used to determine how closely a specific CDF fits the empirical distribution of a given data set. We provide two applications to two real data sets to prove the importance and flexibility of the new model. We compare the fit of the new model with competitve models namely: The Burr X Lomax (BrXLx): $F_{B r X L x}^{(\theta, \alpha, \beta)}(x)=\left[1-\exp \left(-\left\{\left[1+\left(x \beta^{-1}\right)\right]^{\alpha}-1\right\}^{2}\right)\right]^{\theta}$. 
exp-Lx model (Gupta et al. (1998)): $F_{E L x}^{(\alpha, \beta, \lambda)}(x)=\left[1-\left(1+x \beta^{-1}\right)^{-\lambda}\right]^{\alpha}$,

the gamma Lomax (GLx) model (Cordeiro et al. (2015)): $F_{G L x}^{(\alpha, \beta, \lambda)}(x)=\Gamma^{-1}(\alpha) \Gamma\left(\alpha ; \lambda \log \left[1+x \beta^{-1}\right]\right)$, the beta Lomax (BLX) model (Lemonte and Cordeiro (2013)): $F_{B L x}^{(\alpha, \beta, \theta, \lambda)}(x)=\frac{1}{B(\alpha, \theta)} B\left(1-\left(1+x \beta^{-1}\right)^{-\lambda} ; \alpha, \theta\right)$, and Lx model (for $x>0$ and $\alpha, \beta, \lambda, a>0$ ), where $\Gamma(\cdot)$ is the gamma function, $\Gamma(\because ;)$ is the incomplete gamma function, $B(\cdot$,$) is the complete beta function and B(\because, \cdot)$ is the incomplete beta function. The MLEs and the corresponding standard errors (in parentheses) of the model parameters are given in Tables 1 and 3. The numerical values of the statistics $W^{*}$ and $A^{*}$ are listed in Tables 2 and 4 . The histograms of the two data sets and the estimated PDF of the proposed model are displayed in Figures 3 and 4. Many useful Lomax extensions can be used in modeling as a competitive models (see Brito et al. (2017), Elbiely and Yousof (2018), Altun et al. (2018), Korkmaz et al. (2018a,b and 2019), Cordeiro et al. (2018), Elsayed and Yousof (2019), Gad et al. (2019), Yousof et al. (2018 and 2019a,b), Goual and Yousof (2019), Nascimento et al. (2019), Ibrahim and Yousof (2020), Mansour et al. (2020) and Yadav et al. (2020)).

\subsection{Application 1: modeling failure times}

This data set represents the data on failure times of 84 aircraft windshield given in Murthy et al. (2004). The data are: $0.040,1.866,2.385,3.443,0.301,1.876,2.481,3.467,0.309,1.899,2.610,3.478,0.557,1.911,2.625,4.570,1.652$, 2.300, 3.344, 4.602, 1.757, 3.578, 0.943, 1.912, 2.632, 3.595, 1.070, 1.914, 2.646, 3.699, 1.124, 1.981, 2.661, 3.779, $1.248,2.010,2.224,3.117,4.485,1.652,2.229,3.166,2.688,3.924,1.281,2.038,2.823,4.035,1.281,2.085,2.890$, $4.121,1.303,2.089,2.902,4.167,1.432,4.376,1.615,2.223,3.114,4.449,1.619,2.097,2.934,4.240,1.480,2.135$, 2.962, 4.255, 1.505, 2.154, 2.964, 4.278, 1.506, 2.190, 3.000, 4.305, 1.568, 2.194, 3.103, 2.324, 3.376, 4.663. From table 2 and figure 3, the ZTPBrXLx lifetime model is much better than gamma Lomax, beta Lomax, exponentiated Lomax and Lomax models so the exponentiated Lomax, model is a good alternative to these models in modeling aircraft windshield data.

Table 1: MLEs (standard errors in parentheses) for data set $\mathbf{I}$.

\begin{tabular}{ccccc}
\hline Model & \multicolumn{5}{c}{ Estimates } \\
\hline $\operatorname{ZTPBrXLx}(\lambda, \theta, \alpha, \beta)$ & -5.2837 & 0.2860 & 1.553 & 4.6136 \\
& $(93.99)$ & $(0.3119)$ & $(1.395)$ & $(5.843)$ \\
\cline { 2 - 5 } $\operatorname{BLx}(\alpha, \beta, \theta, \lambda)$ & 3.6036 & 118.837 & 33.6387 & 4.8307 \\
& $(0.6187)$ & $(63.715)$ & $(9.2382)$ & $(429.0)$ \\
\cline { 2 - 5 } $\operatorname{BrXLx}(\beta, \theta, \alpha)$ & $5.3 \times 10^{5}$ & $8.2 \times 10^{-1}$ & $1.2 \times 10^{5}$ & \\
& $(0.000)$ & $(0.1051)$ & $(5446.8)$ & \\
$\exp -\operatorname{Lx}(\alpha, \beta, \lambda)$ & 3.6261 & 26257.7 & 20074.5 & \\
& $(0.624)$ & $(99.742)$ & $(2041.8)$ & \\
$\operatorname{GLx}(\alpha, \beta, \lambda)$ & 3.5876 & 37029 & 52001 & \\
& $(0.5133)$ & $(81.16)$ & $(7955)$ & \\
$\operatorname{Lx}(\alpha, \beta)$ & 131789 & 51425 & & \\
& $(296.12)$ & $(5933.5)$ & & \\
\hline
\end{tabular}

Table 2: $W^{*}$ and $A^{*}$ for data set $\mathbf{I}$.

\begin{tabular}{ccc}
\hline Model & $W^{*}$ & $A^{*}$ \\
\hline ZTPBrXLx $(\lambda, \theta, \alpha, \beta)$ & $\mathbf{0 . 0 7 4 6}$ & $\mathbf{0 . 1 5 3 1}$ \\
$\operatorname{BrXLx}(\beta, \theta, \alpha)$ & 0.0764 & 0.5844 \\
$\operatorname{BLx}(\alpha, \beta, \theta, \lambda)$ & 1.4084 & 0.1680 \\
$\exp -\operatorname{Lx}(\alpha, \beta, \lambda)$ & 1.7435 & 0.2194 \\
$\operatorname{GLx}(\alpha, \beta, \lambda)$ & 1.3667 & 0.1619 \\
$\operatorname{Lx}(\alpha, \beta)$ & 1.3976 & 0.1665 \\
\hline
\end{tabular}

\subsection{Application 2: modeling service times}

This real data set represents the data on service times of 63 aircraft windshield given in Murthy et al. (2004). The data are: $0.046,1.436,2.592,0.140,1.492,2.600,0.150,1.580,2.670,0.248,1.7190,2.717,0.2800,1.794,2.819,0.3130$, $1.915,2.820,0.389,1.9200,2.878,0.487,1.9630,2.950,0.622,1.978,3.0030,0.9000,2.053,3.1020,0.952,2.065$, 3.3040, 0.9960, 2.117, 3.483, 1.0030, 2.137, 3.500, 1.0100, 2.141, 3.6220, 1.085, 2.163, 3.6650, 1.092, 2.183, 3.695, $1.1520,2.2400,4.015,1.183,2.3410,4.628,1.2440,2.435,4.806,1.249,2.4640,4.881,1.262,2.5430,5.140$. From 
table 4 and figure 4, the ZTPBrXLx lifetime model is much better than gamma Lomax, beta Lomax, exponentiated Lomax and Lomax models so the new model is a good alternative to these models in modeling the service times data.

Table 3: MLEs (standard errors in parentheses) for data set II.

\begin{tabular}{ccccc}
\hline Model & \multicolumn{4}{c}{ Estimates } \\
\hline $\operatorname{ZTPBrXLx}(\lambda, \theta, \alpha, \beta)$ & -1.4557 & 0.4652 & 1.3517 & 3.9449 \\
& $(2.3026)$ & $(0.215)$ & $(91.50)$ & $(6.440)$ \\
\cline { 2 - 5 } $\operatorname{BLx}(\alpha, \beta, \theta, \lambda)$ & 1.9218 & 169.58 & 31.2595 & 4.9685 \\
& $(0.3185)$ & $(339.21)$ & $(316.8)$ & $(50.528)$ \\
\cline { 2 - 5 } $\operatorname{BrXLx}(\beta, \theta, \alpha)$ & 0.6467 & 0.5987 & 1.6211 & \\
& $(0.048)$ & $(0.390)$ & $(0.959)$ & \\
$\exp -\operatorname{Lx}(\alpha, \beta, \lambda)$ & 1.9145 & 32882 & 22971 & \\
& $(0.3483)$ & $(162.22)$ & $(3209.5)$ & \\
$\operatorname{GLx}(\alpha, \beta, \lambda)$ & 1.9073 & 39197.6 & 35842.4 & \\
& $(0.3214)$ & $(151.65)$ & $(6945)$ & \\
$\operatorname{Lx}(\alpha, \beta)$ & 207019 & 99269 & & \\
& $(301.24)$ & $(11863)$ & & \\
\end{tabular}

Table 2: $W^{*}$ and $A^{*}$ for data set II.

\begin{tabular}{ccc}
\hline Model & $W^{*}$ & $A^{*}$ \\
\hline $\operatorname{ZTPBrXLx}(\lambda, \theta, \alpha, \beta)$ & $\mathbf{0 . 0 3 3 7}$ & $\mathbf{0 . 1 2 2 9 5}$ \\
$\operatorname{BrXLx}(\beta, \theta, \alpha)$ & 0.0876 & 0.5278 \\
$\operatorname{BLx}(\alpha, \beta, \theta, \lambda)$ & 1.1336 & 0.1872 \\
$\operatorname{ELx}(\alpha, \beta, \lambda)$ & 1.2331 & 0.2037 \\
$\operatorname{GLx}(\alpha, \beta, \lambda)$ & 1.1121 & 0.2038 \\
$\operatorname{Lx}(\alpha, \beta)$ & 1.1265 & 0.1861 \\
\hline
\end{tabular}

\section{Concluding remarks}

The main goal of this article is to introduce a new extension of the continuous Lomax distribution with a strong physical motivation. Some of its statistical properties such as moments, incomplete moments, moment generating function, quantile function, random number generation, quantile spread ordering and moment of the reversed residual life are derived. Two applications are provided to illustrate the importance and flexibility of the new model. The new lifetime model is much better than other competitive models such as the gamma Lomax, the Burr X Lomax, the beta Lomax, the exponentiated Lomax and the original Lomax model so the new model is a useful alternative to these models in modeling the failure and service times data.

\section{References}

1. Abouelmagd, T. H. M. (2018). A new flexible version of the Lomax distribution with applications. International Journal of Statistics and Probability, 7(5), 120-132.

2. Altun, E., Yousof, H. M., Chakraborty, S. and Handique, L. (2018). Zografos-Balakrishnan Burr XII distribution: regression modeling and applications. International Journal of Mathematics and Statistics, 19(3), 46-70.

3. Aryal, G. R. and Yousof, H. M. (2017). The exponentiated generalized-G Poisson family of distributions. Stochastics and Quality Control, 32(1), 7-23.

4. Brito, E., Cordeiro, G. M., Yousof, H. M., Alizadeh, M. and Silva, G. O. (2017). Topp-Leone Odd Log-Logistic Family of Distributions, Journal of Statistical Computation and Simulation, 87(15), 3040-3058.

5. Cordeiro, G. M., Ortega, E. M. and Popovic, B. V. (2015). The gamma-Lomax distribution. Journal of Statistical computation and Simulation, 85(2), 305-319.

6. Cordeiro, G. M., Yousof, H. M., Ramires, T. G. and Ortega, E. M. M. (2018). The Burr XII system of densities: properties, regression model and applications. Journal of Statistical Computation and Simulation, 88(3), $432-456$.

7. Elbiely, M. M. and Yousof, H. M. (2018). A New Extension of the Lomax Distribution and its Applications, Journal of Statistics and Applications, 2(1), 18-34.

8. Elsayed, H. A. and Yousof, H. M. (2019). A new Lomax distribution for modeling survival times and taxes revenue data sets. Journal of Statistics and Applications, forthcoming.

9. Gad, A. M., Hamedani, G. G., Salehabadi, S. M. and Yousof, H. M. (2019). The Burr XII-Burr XII distribution: 
mathematical properties and characterizations. Pakistan Journal of Statistics, 35(3), 229-248.

10. Goual, H. and Yousof, H. M. (2019). Validation of Burr XII inverse Rayleigh model via a modified chi-squared goodness-of-fit test. Journal of Applied Statistics, 47(1), 1-32.

11. Gupta, R. C., Gupta, P. L. and Gupta, R. D. (1998). Modeling failure time data by Lehman alternatives. Communications in Statistics-Theory and methods, 27(4), 887-904.

12. Ibrahim, M. and Yousof, H. M. (2020). A new generalized Lomax model: statistical properties and applications. Journal of Data Science, 18(1), 190-217.

13. Korkmaz, M. C., Altun, E., Yousof, H. M. and Hamedani G. G. (2019). The odd power Lindley generator of probability distributions: properties, characterizations and regression modeling, International Journal of Statistics and Probability, 8(2), 70-89.

14. Korkmaz, M. C., Yousof, H. M., Hamedani G. G. and Ali, M. M. (2018a). The Marshall-Olkin generalized G Poisson family of distributions, Pakistan Journal of Statistics, 34(3), 251-267.

15. Korkmaz, M. C. Yousof, H. M., Rasekhi, M. and Hamedani G. G. (2018b). Bayesian analysis, classical inference and characterizations for the odd Lindley Burr XII model. Mathematics and Computers in Simulation. Journal of Data Science, 16(2), 327-354.

16. Lemonte, A. J. and Cordeiro, G. M. (2013). An extended Lomax distribution. Statistics, 47(4), 800-816.

17. Lomax, K.S. (1954). Business failures: Another example of the analysis of failure data, Journal of the American Statistical Association 49, 847-852.

18. Mansour, M., Yousof, H. M., Shehata, W. A. and Ibrahim, M. (2020). A new two parameter Burr XII distribution: properties, copula, different estimation methods and modeling acute bone cancer data. Journal of Nonlinear Science and Applications, forthcoming.

19. Murthy, D.N.P. Xie, M. and Jiang, R. (2004). Weibull Models, Wiley.

20. Ramos, M. W. A. Marinho, P. R. D. Cordeiro, G. M. da Silva, R. V. and Hamedani, G. G. (2015). The Kumaraswamy-G Poisson family of distributions, J. Stat. Theory Appl. 14, 222-239.

21. Nascimento, A. D., Silva, K. F., Cordeiro, G. M., Alizadeh, M., Yousof, H. M. and Hamedani, G. G. (2019). The odd Nadarajah-Haghighi family of distributions: properties and applications. Studia Scientiarum Mathematicarum Hungarica, 56(2), 185-210.

22. Sen, S., Korkmaz, M. C. and Yousof, H. M. (2018). The quasi xgamma-Poisson distribution. Theory and Applications of Statistics and Information, 18(3), 65-76.

23. Yadav, A. S., Goual, H., Alotaibi, R. M., Ali, M. M. and Yousof, H. M. (2020). Validation of the Topp-LeoneLomax model via a modified Nikulin-Rao-Robson goodness-of-fit test with different methods of estimation. Symmetry, 12(1), 57.

24. Yousof, H. M., Afify, A. Z., Hamedani, G. G. and Aryal, G. (2017). The Burr X generator of distributions for lifetime data. Journal of Statistical Theory and Applications, 16, 288-305.

25. Yousof, H. M., Altun, E., Rasekhi, M., Alizadeh, M., Hamedani, G. G. and Ali, M. M. (2019a). A new lifetime model with regression models, characterizations and applications. Communications in Statistics-Simulation and Computation, 48(1), 264-286.

26. Yousof, H. M., Ahsanullah, M. and Khalil, M. G. (2019b). A New Zero-Truncated Version of the Poisson Burr XII Distribution: Characterizations and Properties. Journal of Statistical Theory and Applications, 18(1), 1-11.

27. Yousof, H. M., Majumder, M., Jahanshahi, S. M. A., Ali, M. M. and Hamedani G. G. (2018). A new Weibull class of distributions: theory, characterizations and applications, Journal of Statistical Research of Iran, 15, 4583.

28. Yousof, H. M., Mansoor, M., Alizadeh, M., Afify, A. and Ghosh, I. (2020). The Weibull-G Poisson family for analyzing lifetime data. Pakistan Journal of Statistics and Operation Research, 131-148. 


\section{Appendix}
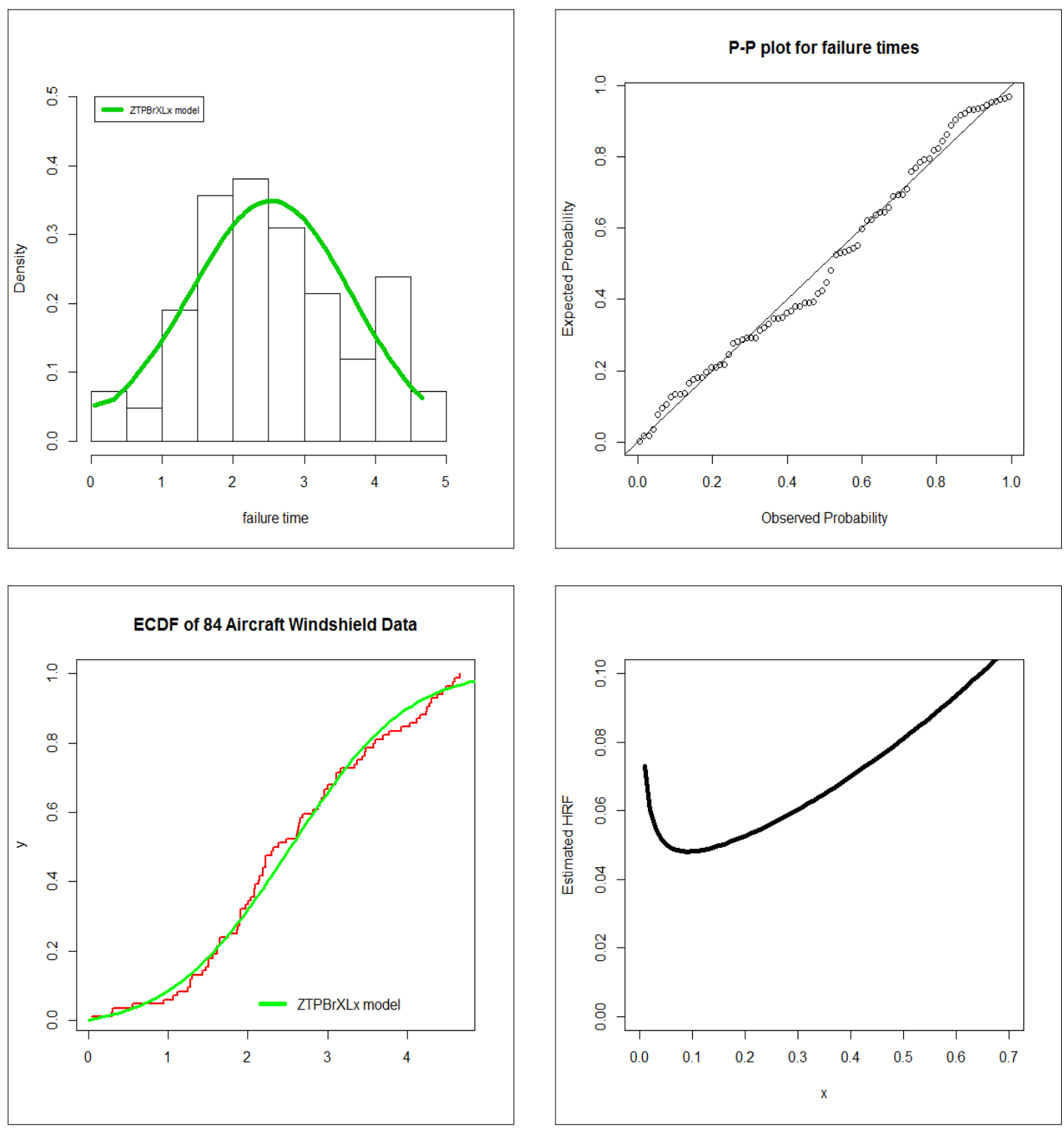

Figure 3: Estimated PDF, P-P plot, Estimated CDF, HRF for data set I. 

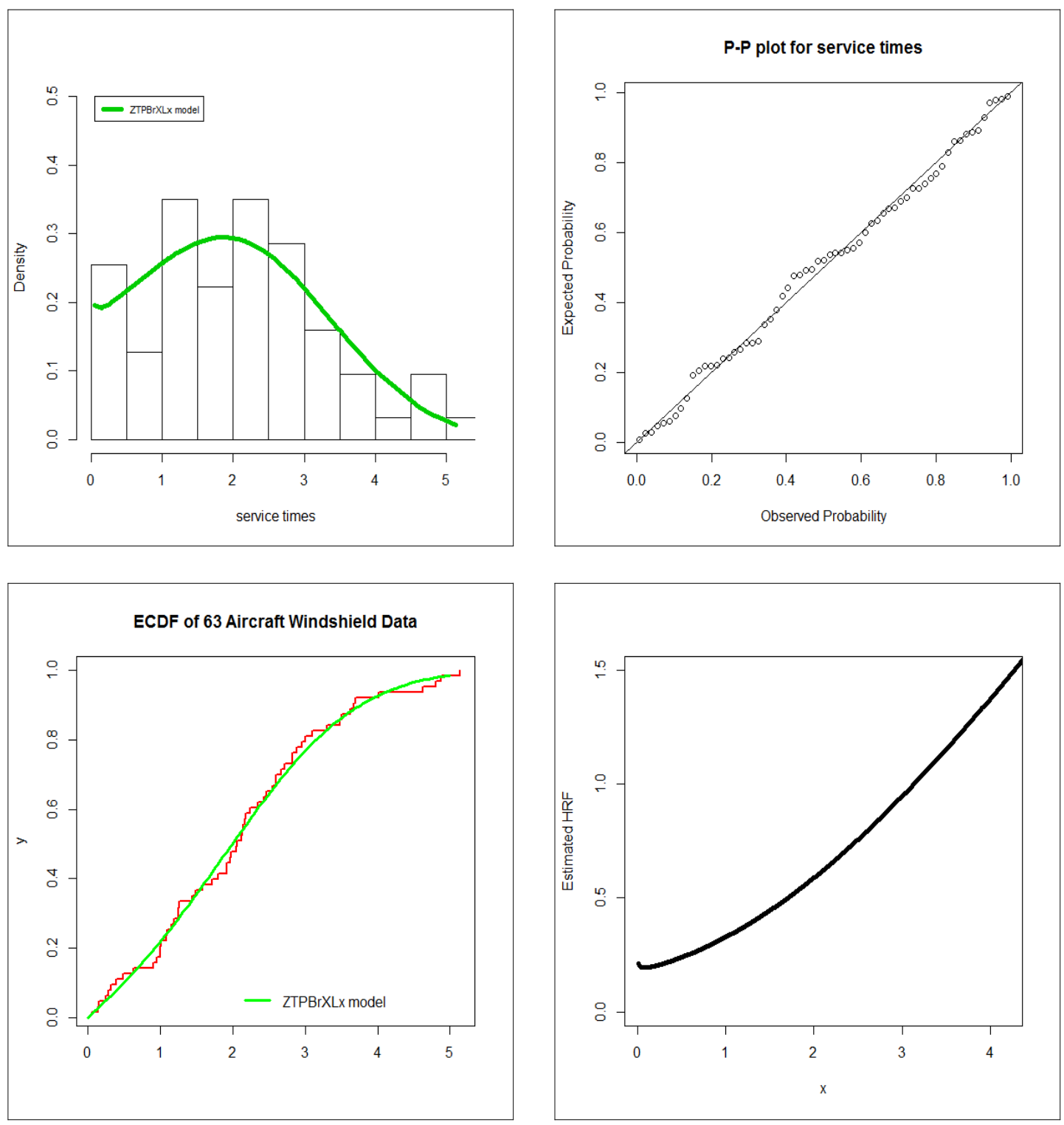

Figure 4: Estimated PDF, P-P plot, Estimated CDF, HRF for data set II. 